\title{
C-REACTIVE PROTEIN LEVELS - INDICATOR FOR PROGNOSIS OF SPONTANEOUS PRETERM BIRTH IN BULGARIAN WOMEN

\author{
Iliana Koleva-Korkelia ${ }^{1}$, Yanka Karamalakova ${ }^{2}$
}

\begin{abstract}
It is estimated that every year fifteen million premature babies are born worldwide mainly due to spontaneous preterm birth (sPTB). Furthermore, in clinical settings, there still are no reliable and accurate tools to predict preterm labor. Hence, the aim of this pioneering research was to estimate the relationship between the maternal inflammatory indicator and sPTB in a case-control study between 220 South Bulgarian women. The study was conducted at UMBAL, Stara Zagora, Bulgaria (2017-2020) and enrolled a total of 220 women, determined into two groups: 1) TB $(n=110)$, who were to give birth at term $\geq 37$ to $\leq 39+6$ gestation weeks with active labor at the time of hospitalization; and 2$)$ sPTB $(n=110)$, women with preterm birth $\leq 32-34+6$ gestation weeks and declared active labor, who were to give birth within 5-24 hrs. The inflammatory indicators/CRP concentration was quantified in plasma by immunoturbidimetric methods within $2 \mathrm{hrs}$. in $\mathrm{mg} / \mathrm{l}$. The median maternal CRP $(8.77 \pm 3.91)$, with cutoff $=4.9 \mathrm{mg} / \mathrm{l}$ was identified as optimal inflammation with highest risk of sPTB (sensitivity $=86.6 \%$; specificity $=53.7 \%, \mathrm{p}<0.0001$ ). Moreover, a cutoff CRP $=4.9 \mathrm{mg} / \mathrm{l}$ was found to be most effective in determining maternal age $\leq 19$ years, the sensitivity of $68.6 \%$, and positively correlated $\mathrm{OR}=8.122$ vs. OR $=$ 2.354 , with increased total sPTB risk at $\leq 32-34+6$ weeks, respectively $(\mathrm{p}<0.001)$.

In conclusion, increased CRP concentrations and a decreased maternal age were associated with increased risks of sPTB, before $\leq 32-34+6$ weeks. Minimal inflammation and other factors in combination may also act as sPTB prognosis.
\end{abstract}

UDC Classification: 618, DOI: https://doi.org/10.12955/pmp.v2.177

Keywords: CRP, preterm birth, term birth, inflammation

\section{Introduction}

Spontaneous preterm birth (sPTB) remains a socially significant problem globally just as in Bulgaria. According to a 2012 report by the World Health Organization, each year, about 15 million premature babies are born worldwide. In Europe, these births can range to around 500,000. Besides, 1.1 million children are unable to survive due to the complications related with premature birth. Rogers and Velton (2011) comment on the high rates of mental, physical, and neurological complications in PTB children.

Premature births are categorized into two major types depending on their main cause: 1) Idiopathic spontaneous premature birth (70-80\%); there is declared labor with preserved integrity of the amniotic membranes (40-50\%), or birth begins after premature ejaculation of the amniotic sac without declared labor (25-40\%); 2) Artificially induced (elective) premature birth (20-30\%); it is declared by induction of labor or by caesarean section according to the indications of the mother or fetus before the end of the 37 gestational weeks, as mentioned by Menon (2008). Nevertheless, the reasons for the spontaneous onset of premature birth are not fully understood. In sPTB there is increased uterine contractility, structural changes in the cervix, various processes in the fetal membranes and uterine decidua, changes in hormonal levels (Esplin 2014) of the pregnant woman, etc. and these factors then trigger spontaneous labor resulting in the birth of a premature baby (Menon 2008; Esplin 2014). It is now accepted that childbirth begins under the influence of biochemical and mechanical stimuli generated by the mother and the fetus (Mitchell et al. 2016). The placenta and fetal membranes play a major role in initiating labor. Menon (2019) determines that the fetus can modulate the exact moment of premature birth. According to Menon (2019), the main etiological cause of premature birth is considered to be the ascending bacterial infection of the amniotic membranes, amniotic fluid, placenta, umbilical cord, and fetus. Bacterial infection occurs with reduced local immunity in the vagina and cervical canal of the pregnant woman, or with reduced bactericidal properties of the amniotic fluid which is a kind of a biological barrier between the fetus and the extra fetal structures, as stated by Romero et al. (2015) and Huang et al. (2020). Inflammation is the only mechanism evidenced to lead to premature birth. Liggins G. (1981) links the "maturation" of the cervix to the inflammatory response of the body. Indeed, signs of inflammation are found in the cervix, myometrium, amniotic membranes, and intraamniotic space. Seferovic et al. (2019) specify that infection of the amniotic membranes, water, umbilical cord, and placenta is a known and proven cause of preterm birth.

\footnotetext{
${ }^{1}$ Trakia University, Medical Faculty, Obstetrics and Gynecology Clinic, Stara Zagora, Bulgaria, iliana_mih@abv.bg

${ }^{2}$ Trakia University, Medical Faculty, Chemistry and Biochemistry, Stara Zagora, Bulgaria, ykaramalakova@gmail.com
} 
There is a possibility of "sterile" inflammation in them too, which can be found in cases of premature birth with intact amniotic membranes.

It is an expression of the non-specific defense mechanism in cellular stress, cell death (Almskaar 2019), and even leads to perinatal death. Clinical studies by Ho et al. (2012) and Navolan et al. (2020) record changes of intense concentration in the levels of inflammatory markers and activation of the cellular immune response during intrauterine infection, $\mathrm{sPTB}$, or preeclampsia.

C-reactive protein (CRP) is a systemic, highly sensitive, inflammatory protein that must be tested in this situation. According to Moghaddam Banaem et al. (2012), CRP is a pathogenic and diagnostic factor in the acute phase of inflammation as it is produced in hepatocytes as a response to an ongoing infection or tissue disorder. Guney (2020) found that the release of inflammatory cytokines, including interleukin-1, interleukin-6, and tumor necrotic factor-alpha, stimulate CRP production. Moghaddam Banaem et al. (2012) further emphasize that in previous studies, elevated serum CRP levels were directly associated with intrauterine infection. The registration of local oxidative stress at the site of implantation of the ovum also leads to increased serum CRP concentrations (Diamanti-Kandarakis et al. 2017) during the 4th gestational week of pregnancy. However, in their study, Ferguson et al. (2014) present contradictory results regarding the relationship between inflammatory cytokines, CRP and PTB. Additionally, PTB studies, in most cases, are limited to the first and third trimester only and do not pay attention to the oxidative changes in the CRP concentration at the time of birth. More importantly, the PTB development in southwest Bulgarian women has not been reported yet.

To better understand the changes in maternal autoimmunity during pregnancy and to find a marker for risk observation, we broke new ground by measuring CRP concentrations collected in $\leq 32-34^{+6}$ gestational weeks and by calculating the relationship between plasmatic inflammation and spontaneous PTB in a prospective case-control study in South Bulgarian women.

\section{Patients and Methodology}

\section{Ethical Considerations}

This prospective case-control study was set in June 2017 and is based on the Stara Zagora birth casecontrol study. It was conducted at the Clinic of Obstetrics and Gynecology, University Hospital "Prof. St. Kirkovich" together with the Medicine Faculty, Trakia University, Stara Zagora, Bulgaria. The Scientific Committee at the Faculty of Medicine approved a written informed consent (2017/2020 MF, TrU, Stara Zagora) obtained from the patients after hospitalization for the period of June 2017 and October 2020. The case-control study was then conducted among 220 participants and was followed up until the end of pregnancy. As a result, there were pregnancy outcomes for 220 patients. All participants completed face-to-face interviews and professional maternity checkups always done by the professional gynecologist.

\section{sPTB Registration}

The eligibility criteria involved patients who delivered live singleton spontaneous PTB along the lines of the following specifications: gestational age $\leq 32$ and $\leq 34^{+6}$ gestation weeks; non-smokers; live fetus; preserved amniotic sac; lack of ultrasound and laboratory data for fetal abnormalities; lack of history of systemic disease; lack of severe systemic infection (respiratory, renal and cardiovascular); lack of gestational or previous diabetes; lack of preeclampsia, antepartum uterine bleeding, hypertension, or cancer. The 220 cases (Table 1) were divided into two groups, 18-42, 5 years:

1) TB (controls) $(n=110)$, the women who gave birth with singleton neonate in term $=37$ and $\leq$ $39^{+6}$ weeks; with active labor at the time of hospitalization and preserved integrity of the amniotic sac.

2) $\operatorname{sPTB}(\mathrm{n}=110)$, women with preterm birth $\leq 32-34^{+6}$ gestation weeks; admitted with declared active labor along with preserved integrity of the amniotic sac and gave birth within 24 hours after hospitalization.

The gestational age was calculated on the date of the last regular menstrual period (LMP) and verified by vaginal speculum examination, abdominal ultrasonography for measurement (Aloka, Prosound alpha 6) of AFI. Both groups consumed additional amounts of folic acid ( $\leq 300 \mathrm{mg} /$ day), vitamins $(\leq$ $350 \mathrm{mg} /$ day $)$, and magnesium $(\leq 360 \mathrm{mg} /$ day $)$ during pregnancy. 


\section{Blood Collection and Maternal Plasma CRP Analysis}

The tested blood samples were taken from v. cubitalis in vacutainer with an anticoagulant at the time of hospitalization and were examined within a 2-hour time period. Maternal CRP concentrations were quantified in the blood plasma by a highly sensitive immunoturbidimetric method with a CRP assay kit (Randox Ltd., UK). The method sensitivity was $0.3 \mathrm{mg} / \mathrm{l}$. The following values of the indicator were taken into account when reporting and interpreting the results: $\mathrm{CRP} \geq 5 \mathrm{mg} / \mathrm{l}$, (normal); $\mathrm{CRP}=5$ 7-10 mg/l, (limited); CRP > $10 \mathrm{mg} / \mathrm{l}$, (systemic inflammatory process); CRP > $20 \mathrm{mg} / \mathrm{l}$, (infection).

\section{Data Sources}

The CRP analysis was obtained from the hospital laboratory of UMHAT of the Medical Faculty at Stara Zagora. The data for the pregnant women was exported from the hospital system to minimize the difference in reporting the results.

\section{Statistical Analysis}

All statistical analyses were performed using IBM SPSS 25.0. The continuous variables were performed by Student's test for potential factors [gestational age, diabetes, parity, body mass index (BMI), abortion, cervical length] and ROC analysis categorical variables were used to determine the cut-off point and statistical significance of a quantitative variable. We evaluated the associations between maternal plasmatic CRP concentrations and SPTB by using logistic regression receiving operating characteristic (ROC) curve to determine the best cut-off point for plasmatic CRP. The odd ratios and accuracy (95\% confidence intervals) were calculated to establish the CRP concentrations as a risk factor of spontaneous preterm birth. $\mathrm{p}<0.05$ was considered statistically significant.

\begin{tabular}{|c|c|c|c|}
\hline Characteristics & $\begin{array}{c}\text { TB }(n=110) \\
37-39^{+6} \text { r.c. }\end{array}$ & $\begin{array}{c}\text { sPTB }(n=110) \\
32-34^{+6} \text { r.c. }\end{array}$ & $* \mathbf{p}$ \\
\hline Age (years) & $26.9 \pm 5.8$ & $31.8 \pm 4.12$ & $0.043^{\mathrm{a}, \mathrm{t} \text { test }}$ \\
\hline Family History of Diabetes & None & None & $\leq 0.00$ \\
\hline Body Mass Index - BMI $\left(\mathrm{kg} / \mathrm{m}^{2}\right)$ & $39.6 \pm 2.6$ & $19.3 \pm 0.81$ & $\leq 0.055^{\mathrm{a}, \mathrm{t} \text { tes }}$ \\
\hline Gestational Age (weeks/range) & $38.2 \pm 1.9$ & $32.9 \pm 4.12$ & $\leq 0.004^{\text {a, tes }}$ \\
\hline Eclampsia/Pre-Eclampsia & none & none & - \\
\hline Infectious Diseases & none & none & - \\
\hline Pregnancy Parity & NA - not applicable & NA & - \\
\hline History of Abortion & 0.00 & $0.00(0-1)$ & $\leq 0.00$ \\
\hline \begin{tabular}{|l|} 
Previous SPTB \\
\end{tabular} & 0.00 & $0.00(0-2)$ & $\leq 0.00$ \\
\hline $\begin{array}{l}\text { Cervical Length }(\mathrm{cm}) \\
<2.6 \\
\geq 2.6\end{array}$ & $\begin{array}{l}9(7 \%) \\
101(93 \%)\end{array}$ & $\begin{array}{l}12(6 \%) \\
98(96 \%)\end{array}$ & $\begin{array}{l}\leq 0.001^{\mathrm{a}, \mathrm{t} \text { tes }} \\
\leq 0.002^{\mathrm{a}, \mathrm{tes}}\end{array}$ \\
\hline
\end{tabular}

Source: Author

\section{Results and Discussion}

Over the last decade, studies associated with an increased SPTB risk have stressed on infectious diseases in the vaginal microflora and disorders of gestational tissue and cervicovaginal fluid. Lamont (2015) demonstrates that local or systemic infection is a major factor in the PTB etiology. Also, Lamont (2015) and Menon (2019) comment that as the gestational age progresses in singleton pregnancies, the sPTB infection relationship changes characteristically. Maternal plasmatic CRP concentration is the most commonly used clinical biomarker to predict infectious and inflammatory difficulties in women with sPTB. Nonetheless, the studies of Cetinkaya et al. (2017) and Huang et al. (2020) discuss that the elevated CRP concentrations were linked to sPTB.

\section{Clinical characteristics}

The clinical characteristics of the 220 women included in the study are summarized in Table 1 . The mean gestational age for the patients with $\mathrm{sPTB}(\leq 32.9 \pm 4.12$ weeks, $\mathrm{p} \leq 0.004$, $t$-test $)$ was significantly lower than the mean gestational age $(=38.2 \pm 1.9$ weeks, $\mathrm{p} \leq 0.004, t$-test $)$ for TB controls. According to Lamont $(2015 ; 2019)$, abnormal genital microflora, maternal endometritis, and chorioamnionitis are present in most cases where PTB occurs spontaneously between 26 and 33 completed gestational weeks. No statistically significant clinical signs and paraclinical evidences of infection were found in the preterm births included in our study. This, in turn, does not exclude the 
presence of subclinical intrauterine inflammation which may be the cause of the synthesis and release of proinflammatory cytokines that induce uterine contractions (Lamont, 2019) and subsequent PTB.

The mean age of patients with premature uterine contractions who completed with sPTB was $\leq 31.8 \pm$ 4.12 years ( $\mathrm{p} \leq 0.043$, $t$-test), while the controls mean age was $26.9 \pm 5.8$ years ( $\mathrm{p} \leq 0.043$, $t$-test). We also observed statistically significant differences between body mass indices for the two groups respectively (mean $\leq 19.3 \pm 0.81 \mathrm{sPTB}$ vs. mean $\leq 39.6 \pm 2.6, \mathrm{p} \leq 0.055 \mathrm{~TB}, t$-test). Furthermore, we found a statistically significant relationship between the TB and SPTB groups in all the three measured factors respectively $\left({ }^{\mathrm{a}} \mathrm{p}<0.002, t\right.$-test; ${ }^{\mathrm{a}} \mathrm{p}<0.003, t$-test; ${ }^{\mathrm{a}} \mathrm{p}<0.005, t$-test $)$. Most of the participating women were manual workers, non-smokers, living in a low-income social environment, and were taking folic acid, vitamins and magnesium irregularly during pregnancy. These findings are consistent with previous observations made by D'Silva (2018) and Lamont (2019). The authors found that sPTB was closely associated with low levels of education, low family income, insufficient access to prenatal care, older age, and systemic active or passive smoking. Poor nutritional status, irregular dietary intake, low maternal weight, and low BMI before and during pregnancy are strong predictors of PTB (D'Silva, 2018; Lamont, 2019). The available evidence for an increased BMI relative to SPTB is, however, contradictory. Obesity is associated with an increased risk of miscarriage, as noted by Lashen et al. (2004) and Catov et al. (2008), and in most studies, a high BMI has either a protective effect or no direct correlation with the sPTB onset, as remarked by Hendler et al. (2005) and Catov et al. (2008). Our observation registered that the cervical length (CL) mean in the sPTB group was 1.85 $\mathrm{cm}(\leq 0.001$ vs. controls) which is less than the mean of cervical length of the TB group $2.91 \mathrm{~cm}(\mathrm{p}<$ 0.004). Parallel to our results, Tommaso and Berghella (2013) comment that women with a cervical length $\leq 15 \mathrm{~mm}$ have an approximate 50\% likelihood of early spontaneous PTB $\leq 32$ gestation weeks regardless of other risk factors.

\section{Biochemical analysis of the plasmatic CRP}

Despite a large number of investigations reporting an increase in the CRP concentrations is directly correlated with spontaneous PTB, this study is a pioneering one for reporting the problem in South Bulgarian women. There were no detectable clinical signs of infection in any of the 110 women with sPTB. However, in these SPTB pregnancies, increased CRP concentrations may suggest the presence of subclinical intrauterine infection.

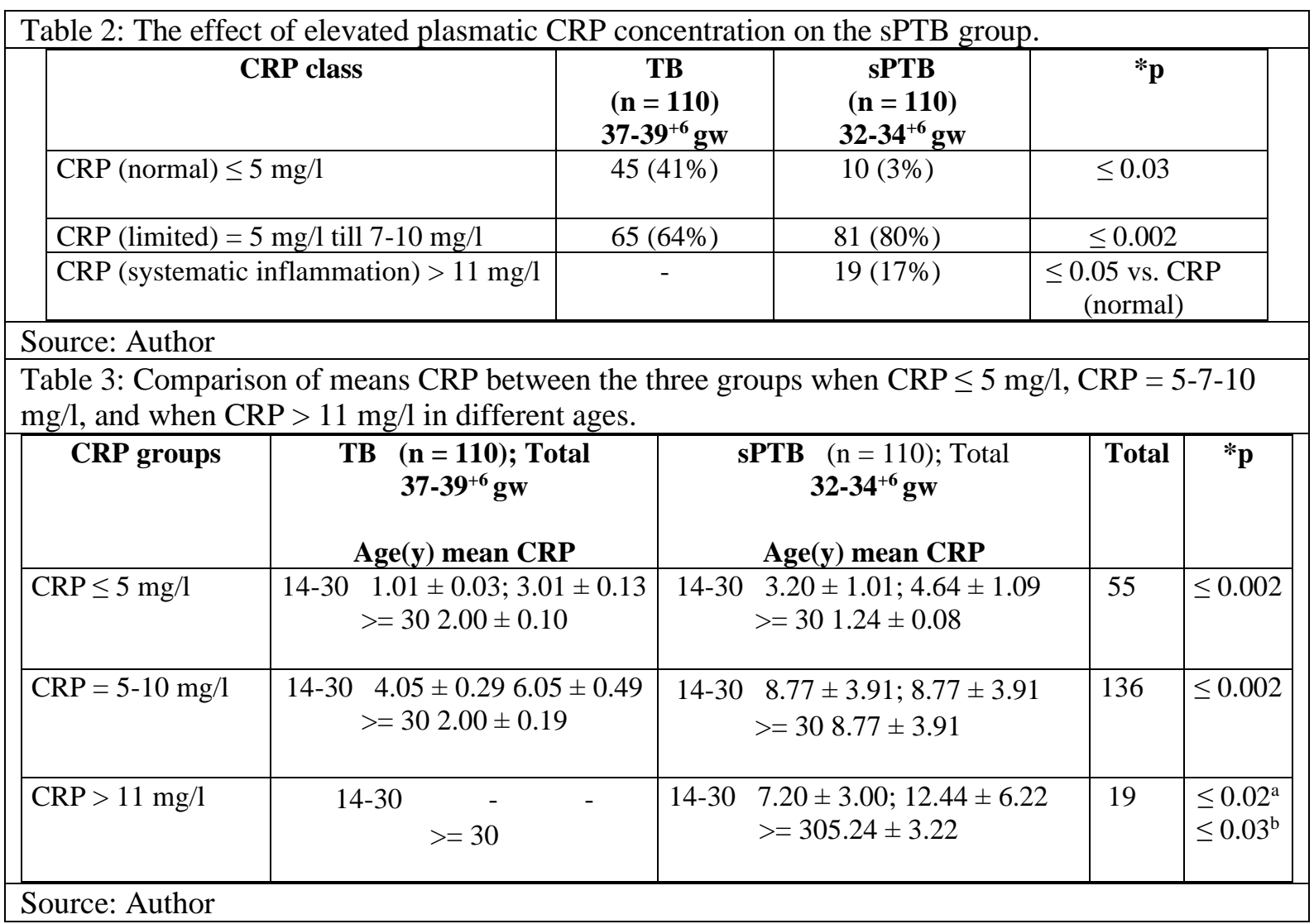


The effect of elevated plasmatic CRP concentrations on the sPTB group is presented in Table 2. It was observed that in $81 \%$ of the sPTB woman with premature uterine contractions, there was a statistically significant increase in CRP limited $(\leq 0.002)$ relative to the CRP concentrations in women who gave birth at term (65\%). Statistically, CRP level elevation and systematic inflammations were reported in $17 \%$ of sPTB patients $(\leq 0.05$ vs. CRP (normal) of TB). There is a significant correlation between the increased CRP concentrations in SPTB as compared to the control group where CRP was elevated above a limited value $=5-10 \mathrm{mg} / \mathrm{l}$. Among the 110 pregnant women included in our investigation who were subject to premature uterine contractions, $81 \%$ of sPTB women delivered preterm within $24 \mathrm{hrs}$. of the beginning of the contractions, and $17 \%$ of sPTB women delivered preterm within $5 \mathrm{hrs}$. of the contractions; $17 \%$ of SPTB women had statistically significant elevation in CRP concentrations (> 11 $\mathrm{mg} / \mathrm{l}, \mathrm{p}<0.05$, t-test). This high percentage of sPTB women $(>90 \%)$ with increased CRP concentrations reflects the production of subclinical infection and increased proinflammatory cytokines. All these factors hypothesized that limited and systematic inflammation processes increased plasmatic CRP, caused preterm delivery at $\leq 32$ gestational weeks, and probably promoted residual oxidative changes in the women. Our results are consistent with those reported by Moghaddam Banaem et al. (2012); the authors found that CRP was higher in women who underwent sPTB before < 34 weeks as compared to those who gave birth after > 37 weeks. In our case-control study, we found that elevated CRP > $5 \mathrm{mg} / \mathrm{l}$ concentration in pregnant women was regarded as high level and pregnant women were at a high risk of spontaneous PTB in association with premature uterine contractions, while CRP > $11 \mathrm{mg} / \mathrm{l}$ concentration was identified as a subgroup of sPTB at the highest risk. In this regard, Dodds and Iams (2003) and Nakishbandy and Barawi (2014) observed that a maternal CRP concentration $\geq 8 \mathrm{mg} / \mathrm{l}$ led to an increased risk of intrauterine infection and $\mathrm{sPTB}$.

The comparison of means CRP between the three groups when CRP $\leq 5 \mathrm{mg} / \mathrm{l},=5-7-10 \mathrm{mg} / \mathrm{l}$, and $>$ $11 \mathrm{mg} / \mathrm{l}$ in the TB and SPTB groups is presented in Table 3. The mean CRP limited concentration in sPTB cases was $8.77 \pm 3.91 \mathrm{mg} / \mathrm{l}$ which is higher than the mean CRP limited concentration in the TB group $(6.05 \pm 0.49, \mathrm{p}<0.005$, t-test $)$. The mean CRP of systematic inflammation in sPTB cases was $12.44 \pm 6.22 \mathrm{mg} / \mathrm{l}$, statistically ten times higher than both, the mean CRP normal values in the TB group (3.01 $\pm 0.13, \mathrm{p}<0.003)$, and the mean CRP limited concentration in the TB group $(6.05 \pm 0.49$, $\mathrm{p}<0.04$ ) (Table 3). Moreover, we found that strongly expressed CRP in limited concentration < 5-10 $\mathrm{mg} / \mathrm{l}$, in systematic inflammations > $11 \mathrm{mg} / \mathrm{l}$, maternal age, and $\mathrm{sPTB} /$ subsequent $\mathrm{PTB}$ were remarkably associated along with the above report, according to the logistic regression analysis.

The relationship between maternal CRP, maternal age, and the risk value of sPTB are shown in Table 4. Compared with the median maternal CRP $(8.77 \pm 3.91)$, a cutoff value of $4.9 \mathrm{mg} / \mathrm{L}$ was identified as optimal concentration for the identification of the highest risk of SPTB with a sensitivity of $86.6 \%$, a specificity of $53.7 \%(0.537)$, a positive predictive value of $60 \%(0.605634)$, a negative predictive value of $83 \%$ (0.833333), a positive likelihood ratio 260.755, and an area under the receiver operating characteristic curve of $68.6 \%(\mathrm{OR}=7.679), \mathrm{p}<0.0001$ (Table 4 , a). The statistically positive correlation between the elevated CRP and the subsequent sPTB suggests that our findings may be due to oxidative disturbances in the maternal organism, i.e., they have an origin different from the typical inflammation. A majority of investigations studied the inflammatory mechanisms of elevated CRP in the first trimester of sPTB and only a few focused on the other oxidative factors that elevated CRP in the late periods of spontaneous birth. In line with our findings, Lucaroni et al (2017) and Ryu et al (2017) confirm that elevated CRP, caused by inflammation or other oxidative cellular changes, may disrupt normal placentation and can have a good diagnostic accuracy in predicting the increased risk in the latent period (5-24 hrs.) of sPTB and PROMP.

Notably, the relationship between plasmatic CRP > $11 \mathrm{mg} / \mathrm{l}$ vs. CRP > $5 \mathrm{mg} / \mathrm{l}$ (Table 4, b) concentrations exhibited a positive $\mathrm{SPTB}$ risk $=2$ with a sensitivity of $16.3 \%$, a specificity of 1.00 , a positive predictive value of 1 , a negative predictive value of $43.8 \%(0.4375)$, a likelihood ratio of $27.3 \%$ (273.344), an accuracy of 49.3\% (0.492958), and an area under the curve of 0.836 (0.693$0.980)$ at a cutoff value of $4.9 \mathrm{mg} / \mathrm{l}$ as a preterm delivery predictive marker $(\mathrm{p}<0.001)$. Although our data provided novel evidence that implied modest increases in CRP, a cutoff value $=4.9 \mathrm{mg} / \mathrm{l}$ was associated with an 8-fold and a 2 -fold increase in the risk of sPTB at $\leq 32-34^{+6}$ gestational weeks. Pitiphat et al (2005) and Catov et al (2007) report a 4.64-fold/12-fold increase in the risk of sPTB at < 34 weeks among women with CRP at $8-12 \mu \mathrm{g} / \mathrm{ml}$, especially those indicated of PTB. 


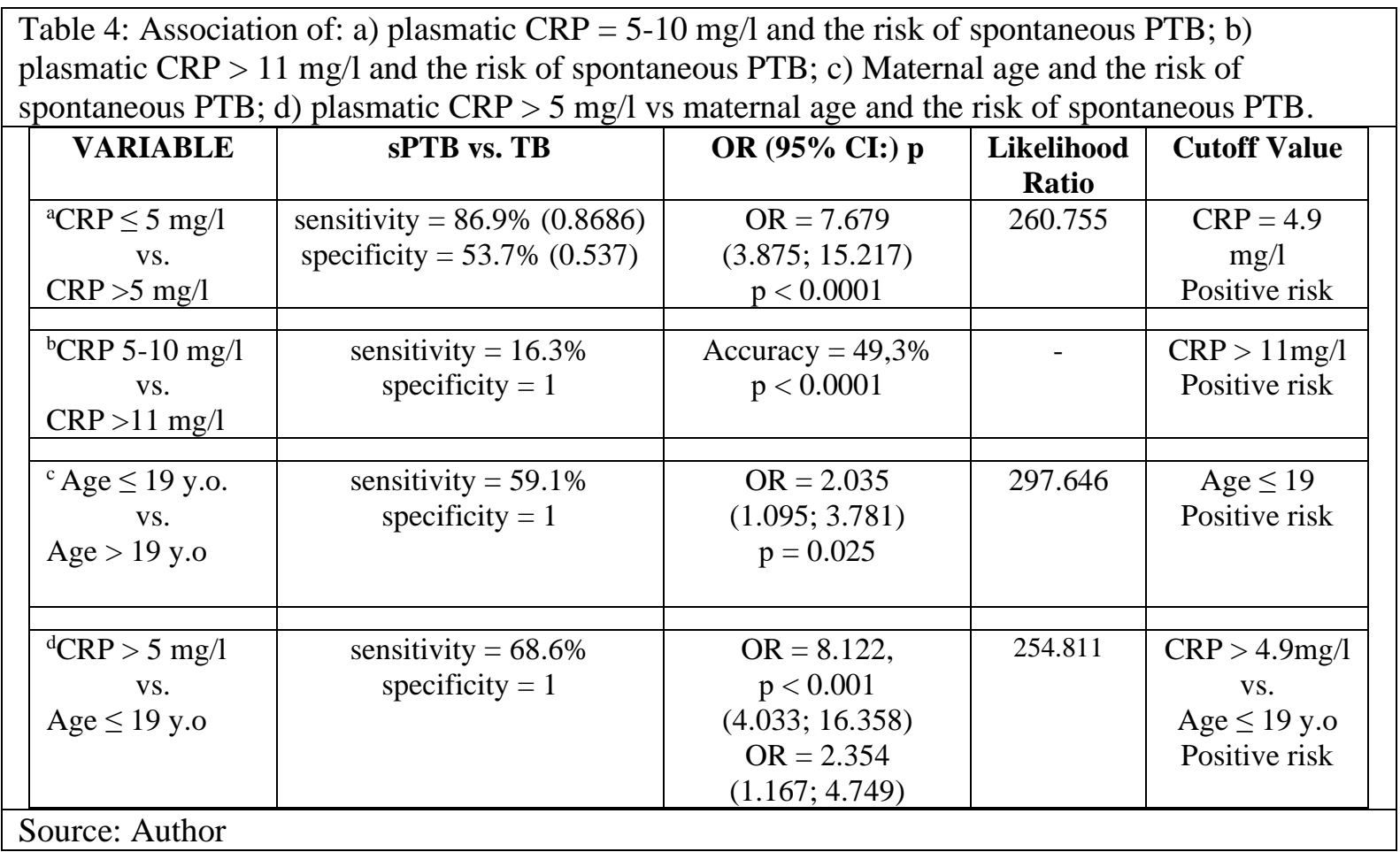

We used a two-component factor analysis to identify the overall risk of sPTB in the gestational age range of $\leq 32$ to $\leq 34^{+6}$ (Table 4, d). Differences in limited inflammatory processes (CRP $>5 \mathrm{mg} / \mathrm{l}-$ factor 1) provide a 4-fold increased risk of sPTB $(\mathrm{p}<0.001)$ even after correction for maternal age. In Addition to this, we observed a cut-off value of CRP $=4.9 \mathrm{mg} / \mathrm{l}$, which was found to be the most effective factor in determining maternal age $\leq 19$ years (factor 2 ) with a sensitivity of 68.6\% (56: 165; $53.7 ; 86.9)$ in correlation to coefficients $\mathrm{OR}=8.122(4.033 ; 16.358)$ for coefficient 1 versus coefficients $\mathrm{OR}=2.354(1.167 ; 4.749)$ for factor 2 , each independently increasing the total risk of $\mathrm{sPTB}$ at $\leq 32$ and $\leq 34^{+6}$ gestational weeks respectively $(\mathrm{p}<0.001)$.

Our study has several strengths. First of all, we only included those women in our study who had a clearly defined spontaneous PTB in the latent period (5-24 hrs.) before birth and term birth without any registered inflammations or history of inflammations. Secondly, maternal plasmatic CRP samples were obtained simultaneously at the time of admission. Thirdly, the study clearly illustrated the link between elevated plasmatic CRP concentrations and decreased maternal age in late periods of preterm birth, and the results were positively correlated. Much noteworthily, the current study is a first of its kind research on spontaneous preterm pregnancy cases in southern Bulgaria, which explores and suggests that at least some oxidative stress metabolic factors, perhaps in combination with minimal inflammation, modified the processes in the maternal organism and may be related to the highest SPTB risk.

\section{Conclusion}

As a conclusion, our case-control study indicates that women with increased CRP $>5 \mathrm{mg} / \mathrm{l}$ concentration have an excess SPTB risk, particularly before $\leq 32$ to $\leq 34^{+6}$ weeks. We have also detected that higher CRP in conjunction with decreased maternal age may be associated with a positive risk suggesting SPTB.

\section{Acknowledgments}

This study was supported by the Ph.D. program of Dr. Iliana Koleva-Korkelia and by scientific project 1/2020 of the Medical Faculty, Trakia University, Bulgaria.

\section{References}

Almskaar, K. (2019). The Placental Microbiome and Preterm Birth: An Evolutionary Life History Perspective (Doctoral dissertation), Libraries. Temple University.

Catov, J. M., Bodnar, L. M., Ness, R. B., Barron, S. J., \& Roberts, J. M. (2007). Inflammation and dyslipidemia related to risk of spontaneous preterm birth. American journal of epidemiology, 166 (11), 1312-1319.

Catov, J. M., Bodnar, L. M., Ness, R. B., Barron, S. J., \& Roberts, J. M. (2008). Inflammation and Dyslipidemia Related to Risk of Spontaneous Preterm Birth. Obstetrical \& Gynecological Survey, 63 (4), 213. 
Cetinkaya, S., Ozaksit, G., Biberoglu, E. H., Oskovi, A., Kirbas, A. (2017). The value of acute phase reactants in predicting preterm delivery. J Matern fetal Neonatal Med. 30 (24): 3004-8.

Di Tommaso, M., \& Berghella, V. (2013). Cervical length for the prediction and prevention of preterm birth. Expert Review of Obstetrics \& Gynecology, 8 (4), 345-355.

Diamanti-Kandarakis, E., Papalou, O., Kandaraki, E. A., \& Kassi, G. (2017). Nutrition as a mediator of oxidative stress in metabolic and reproductive disorders in women. Eur. J. Endocrinol., 176, R79-R99.

Dodds, W. G., Lams, J. D. (2003). Maternal C-reactive protein and preterm labor. J Repord Med.; 32: 527-530.

D'Silva, A. M. (2018). Identification of first trimester maternal serum markers predictive of spontaneous preterm birth. Western Sydney University, Australia.

Esplin, M. S. (2014). Overview of spontaneous preterm birth: a complex and multifactorial phenotype. Clinical obstetrics and gynecology, 57 (3), 518-530.

Ferguson, K. K., McElrath, T. F., Chen, Y. H., Mukherjee, B., \& Meeker, J. D. (2014). Longitudinal profiling of inflammatory cytokines and C-reactive protein during uncomplicated and preterm pregnancy. American journal of reproductive immunology, 72 (3), 326-336.

Guney, G., Taskin, M. I., \& Tokmak, A. (2020). Increase of circulating inflammatory molecules in preeclampsia, an update. European Cytokine Network, 31, 18-31.

Hendler, I., Goldenberg, R. L., Mercer, B. M., et al. (2005). The Preterm Prediction study: association between maternal body mass index and spontaneous and indicated preterm birth, Am J Obstet Gynecol, vol. 192, pp. 882-6.

Ho, M., Faye-Petersen, O. M., Goldenberg, R. L., Carlo, W. A., Cliver, S. P., \& Andrews, W. W. (2012). Elevated midtrimester $\alpha$-fetoprotein and delivery markers of inflammation in a preterm population. The Journal of Maternal-Fetal \& Neonatal Medicine, 25 (11), 2424-2427.

Huang, S., Tian, J., Liu, C., Long, Y., Cao, D., Wei, L., Zhu, X., Tang, R., Liu, W., Zeng, D. and Li, M. (2020). Elevated Creactive protein and complement $\mathrm{C} 3$ levels are associated with preterm birth: a nested case-control study in Chinese women. BMC pregnancy and childbirth, 20 (1), 1-9.

Lamont, R. F. (2015). Advances in the prevention of infection-related preterm birth. Frontiers in immunology, 6, 566.

Lamont, R. F. (2019). Spontaneous preterm labour that leads to preterm birth: An update and personal reflection. Placenta, 79, 21-29.

Lashen, H., Fear, K., \& Sturdee, D. W. (2004). Obesity is associated with increased risk of first trimester and recurrent miscarriage: matched case-control study. Human reproduction, 19 (7), 1644-1646.

Liggins, G. C. (1981). Cervical ripening as an inflammatory reaction. The cervix in pregnancy and labor. Clinical and biochemical investigation, 1-9.

Lucaroni, F., Morciano, L., Rizzo, G., D’Antonio, F., Buonuomo, E., Palombi, L., \& Arduini, D. (2018). Biomarkers for predicting spontaneous preterm birth: an umbrella systematic review. The Journal of Maternal-Fetal \& Neonatal Medicine, 31 (6), 726-734.

Menon, R. (2008). Spontaneous preterm birth, a clinical dilemma: etiologic, pathophysiologic and genetic heterogeneities and racial disparity. Acta obstetricia et gynecologica Scandinavica, 87 (6), 590-600.

Menon, R. (2019). Initiation of human parturition: signaling from senescent fetal tissues via extracellular vesicle mediated paracrine mechanism. Obstetrics \& gynecology science, 62 (4), 199.

Mitchell, M. D., Rice, G. E., Vaswani, K., Kvaskoff, D., \& Peiris, H. N. (2016). Differential regulation of eicosanoid and endocannabinoid production by inflammatory mediators in human choriodecidua. PloS one, 11 (2), e0148306.

Moghaddam Banaem, L., Mohamadi, B., Asghari Jaafarabadi, M., \& Aliyan Moghadam, N. (2012). Maternal serum Creactive protein in early pregnancy and occurrence of preterm premature rupture of membranes and preterm birth. Journal of Obstetrics and Gynaecology Research, 38 (5), 780-786.

Nakishbandy, B. M. N., \& Barawi, S. A. (2014). Level of C-reactive protein as an indicator for prognosis of premature uterine contractions. Journal of prenatal medicine, 8 (1-2), 25.

Navolan, D. B., Stoian, D. L., Bohiltea, R. E., Crainiceanu, Z., Craina, M. L., Cretu, O., Timar, B., Vladareanu, R., Terness, P., Būrger, F. andNemescu, D. (2020). Comparison of early pregnancy serum concentration of neopterin, neopterin/creatinine ratio, C-reactive protein, and chitotriosidase, in pregnant women with birth at term and spontaneous preterm birth. Experimental and Therapeutic Medicine, 20 (3), 2449-2454.

Pitiphat, W., Gillman, M. W., Joshipura, K. J., Williams, P. L., Douglass, C. W., \& Rich-Edwards, J. W. (2005). Plasma Creactive protein in early pregnancy and preterm delivery. American journal of epidemiology, 162(11), 1108-1113.

Rogers, L. K., \& Velten, M. (2011). Maternal inflammation, growth retardation, and preterm birth: insights into adult cardiovascular disease. Life Sci., 89 (13-14): 417-21.

Romero, R., Miranda, J., Chaemsaithong, P., Chaiworapongsa, T., Kusanovic, J. P., Dong, Z., Ahmed, A.I., Shaman, M., Lannaman, K., Yoon, B.H. and Kim, Y. M. (2015). Sterile and microbial-associated intra-amniotic inflammation in preterm prelabor rupture of membranes. The Journal of Maternal-Fetal \& Neonatal Medicine, 28 (12), 1394-1409.

Ryu, H. K., Moon, J. H., Heo, H. J., Kim, J. W., \& Kim, Y. H. (2017). Maternal c-reactive protein and oxidative stress markers as predictors of delivery latency in patients experiencing preterm premature rupture of membranes. International Journal of Gynecology \& Obstetrics, 136 (2), 145-150.

Seferovic, M. D., Pace, R. M., Carroll, M., Belfort, B., Major, A. M., Chu, D. M., Racusin, D.A., Castro, E.C., Muldrew, K.L., Versalovic, J. andAagaard, K. M. (2019). Visualization of microbes by $16 \mathrm{~S}$ in situ hybridization in term and preterm placentas without intraamniotic infection. American journal of obstetrics and gynecology, 221 (2), 146-e1. 